\title{
INVESTIGACIONES
}

\section{El liderazgo compartido como factor de sostenibilidad del proyecto de comunidades de aprendizajes*}

\author{
Shared leadership as a factor of sustainability of project learning communities
}

\author{
Pablo Castillo Armijo, ${ }^{a}$ Ignasi Puigdellivol Aguadé, ${ }^{b}$ Serafín Antúnez Marcos ${ }^{c}$ \\ ${ }^{a}$ Universidad de Valparaíso, Facultad de Humanidades, Valparaíso, Chile \\ Telf.: (56) 953635268. Correo electrónico: pablo.castilloar@uv.cl \\ bUniversitat de Barcelona, Máster de Educación Inclusiva, Barcelona, España \\ Telf.: (34) 934037224. Correo electrónico: ipuigdellivol@ub.edu \\ 'Universitat de Barcelona, Máster en Dirección y Gestión de Centros Educativos, Barcelona, España \\ Telf.: (34) 934035046. Correo electrónico: santunez@ub.edu
}

\begin{abstract}
El presente artículo indaga sobre los significados y valoraciones que los miembros de una Comunidad de Aprendizaje le atribuyen al fenómeno del liderazgo. Utilizamos una metodología cualitativa de estudio de casos, desde una perspectiva comunicativo-crítica. A pesar de las diversas conceptualizaciones, existe una alta valoración sobre la importancia que tiene el liderazgo como base de la mejora y cambio educativo. Nuestro estudio pone de manifiesto también que la sostenibilidad del proyecto depende de la participación y corresponsabilidad en el aprendizaje de todos sus miembros.
\end{abstract}

Palabras clave: administración y organización escolar, liderazgo educativo, comunidades de aprendizaje, aprendizaje activo, innovación educativa.

\section{ABSTRACT}

This article explores the meanings and valuations that members of a learning community attributed the phenomenon of leadership. We used a qualitative case study methodology from a communicative-critical perspective. Despite the various conceptualizations, there is a high value related to the importance of leadership as a basis for improvement and educational change. Our study also shows that the project sustainability depends on the participation and joint responsibility in the learning of all its members.

Key words: educational administration, educational leadership, learning communities, active learning, educational innovation.

Beca de Doctorado en el Extranjero, Segunda Convocatoria año 2009. Programa Becas-Chile CONICYT. 


\section{ANTECEDENTES, JUSTIFICACIÓN Y FORMULACIÓN DEL PROBLEMA}

El estudio nace a partir de la inquietud de indagar en proyectos de cambio y mejora educativos (Hargreaves \& Fink, 2006), en especial de aquellos que la comunidad científica internacional ha reconocido como exitosos en la obtención de resultados de aprendizajes y de mayor inclusión social.

Es así que nos insertamos de voluntarios al interior de una escuela pública española que desarrolla el proyecto educativo de Comunidades de Aprendizaje (CCA), que tiene la particularidad de promover la participación de otros actores en los procesos de enseñanza y aprendizaje y, por tanto, cambiar la gestión y organización interna del centro.

Bajo este contexto es que nos preguntamos sobre las nuevas formas de liderar que tendría esta escuela, partiendo de la evidencia teórica que nos señala al liderazgo pedagógico como el segundo factor en la mejora educativa, luego de las actuaciones del profesor en el aula (OCDE, 2009).

Nuestras preguntas de investigación fueron las siguientes: ¿Qué significados y valoraciones le atribuyen los miembros de la comunidad de aprendizaje al concepto de liderazgo pedagógico?, ¿Es posible identificar capacidades y actitudes específicas del liderazgo en proyectos inclusivos y participativos, como es el caso de Comunidades de Aprendizaje? de ser así ¿Cuáles serían esos perfiles?, ¿Qué influencia tiene el liderazgo en la motivación del centro para emprender y mantener el proyecto de Comunidad de Aprendizaje (CdA)?, ¿Qué componentes formativos deberían introducirse en la formación de los miembros de la CdA que fueran de utilidad para su posible función de liderazgo?

A partir de esas preguntas definimos nuestros objetivos y la estrategia de obtención de información a partir de un estudio de caso cualitativo y orientado por una metodología comunicativa crítica (Gómez et al., 2006) con los actores, es decir, de contraste permanente de las subjetividades surgidas a partir de los datos y las observaciones.

Este estudio correspondió a una investigación desarrollada durante los años 2011 y 2014 al interior de un Centro Público de Educación Infantil y Primaria adscrita al proyecto de CdA ubicada en la comarca de Granollers, Cataluña. Dicha investigación fue llevada cabo en una primera fase como participante voluntario de la escuela y luego en una segunda fase asumiendo un rol de investigador de este contexto particular y que respondía a un estudio de caso (Stake, 1999).

El artículo dará cuenta de un proceso investigativo en profundidad y que devela la complejidad que encierra toda escuela que tiene que luchar para establecer itinerarios de cambio y mejora que sean consensuados por sus miembros, e intentar que sean comprendidos por las administraciones educativas que muchas veces ignoran estos proyectos alternativos y otros logros que no sean los estandarizables y medibles.

\section{REFERENTES TEÓRICOS DEL ESTUDIO}

El aumento de la diversidad socio-cultural en la actual sociedad globalizada, provocado por movimientos migratorios, apertura de fronteras, creación de espacios comunes de intercambio comercial y social, crea nuevos conflictos a los estados en todo orden.

Es así que los retos educativos de fines del siglo XX en relación con la masificación, equidad y calidad educativa comienzan a ser insuficientes de este siglo XXI. Las actuales 
necesidades declaradas por los principales actores educativos (familias, estudiantes, profesores y administradores) transitan hacia la gestión de la diversidad, la resolución de conflictos, la implementación de tecnologías 2.0 en procesos de innovación y creatividad en el aula, la construcción de comunidades efectivas de aprendizaje, en suma, la adquisición de competencias y habilidades específicas requeridas para la actual sociedad del conocimiento.

El diagnóstico educativo y social sobre el papel de la escuela parece ser lo más estudiado en conferencias y foros internacionales ${ }^{1}$ que siguen señalando continuas exclusiones, abandono escolar, desescolarización, situaciones de violación a los derechos fundamentales de las personas y discriminaciones que se desarrollan al interior de algunos centros educativos de estados democráticos.

Estas contradicciones evidentes son parte de la compleja tarea de educar y educarnos en un contexto de mayor complejidad, pero que no nos deben llevar a detener nuestras acciones de transformación que surgen de vez en cuando y que son necesarias de relevar y sostener en el tiempo, porque "El cambio en la educación es fácil de proponer, complicado de llevar a la práctica y extraordinariamente difícil de sostener" (Hargreaves \& Fink, 2008, p. 15).

La ilusión artificial de transformar la educación de manera homogénea en un corto tiempo y de forma descontextualizada debe dar paso a que las experiencias de éxito sean conocidas y socializadas. Los reales cambios pasan primero por la innovación que "Fundamentalmente se centra en la acción educativa que se lleva a cabo en el aula: la innovación puede incidir en varios aspectos, pero especialmente lo hace en los curriculares (contenidos, objetivos, metodologías, evaluación)" (Murillo \& Muñoz-Repiso, 2002, p. 18).

El actual debate educativo debe darse al interior de las aulas y otros espacios de reuniones de aprendizaje como claustros de maestros, de especialistas que apoyan la labor docente, de equipos directivos, de los padres y las madres y reuniones de todas las entidades que colaboran con la escuela a partir de la reflexión, diálogo y cooperación que posibilitarán reales logros educativos.

Los años de las grandes reformas impuestas al sistema educativo deben quedar atrás y dar paso paulatinamente a que las actuales políticas comiencen a reconocer los avances que se dan en la cotidianidad de las aulas, de las escuelas y comunidades que se han decidido a transformar sus realidades.

El cambio educativo no solo debe ser la obtención de resultados medibles y cuantificables, ya no basta hablar de promoción, reprobación o fracaso escolar, sino que hacernos cargo por la integralidad del sujeto que aprende, de su satisfacción y de sus sueños, de sus frustraciones y errores. La escuela como institución formadora de ciudadanía debe ser ese valor agregado que necesita la familia y la sociedad en su conjunto.

El cambio educativo en inclusión y justicia social debe ir de la mano de las políticas públicas de los países, en especial de aquellos que teniendo potencial de crecimiento aún no logran escapar del fantasma del subdesarrollo y la falta de oportunidades de vastos sectores de su población más desfavorecida. Los reales logros en educación necesitan un gran

Cumbre Mundial en favor de la Infancia, Jomtien (1990), la Conferencia sobre el Medio Ambiente y el Desarrollo, Río Janeiro (1992), la Conferencia Mundial de Derechos Humanos, Viena (1993), la Conferencia Mundial sobre Necesidades Educativas Especiales: Acceso y Calidad, Salamanca (1994), la Conferencia Internacional sobre la Población y el Desarrollo, El Cairo (1994), la Cumbre Mundial sobre Desarrollo Social, Copenhague (1995), la Cuarta Conferencia Mundial sobre la Mujer, Beijing (1995), la Reunión de mitad del decenio del Foro Consultivo Internacional sobre Educación para Todos, Ammán (1996), la Conferencia Internacional sobre la Educación de Adultos, Hamburgo (1997), la Conferencia Internacional sobre el Trabajo Infantil, Oslo (1997), el Foro Mundial sobre la Educación para Todos, Dakar (2000) y la Conferencia Internacional de Educación 48ª Reunión: La Educación Inclusiva: Un camino hacia el futuro. Ginebra (2008). 
compromiso de sus actores y especialmente de los sistemas estructurales que administran la educación, en el sentido de prodigar las autonomías necesarias para transformaciones e innovaciones curriculares pedagógicas contextuadas, reflexionadas y de cara a los aprendizajes de todos y todas.

El esfuerzo colectivo, interdisciplinario y encaminado a metas consensuadas entre los actores del proceso, asegurará buenos desempeños laborales y climas de aula que favorezcan nuevos aprendizajes. La gestión de la escuela no debe depender de unos pocos, debe sumar ideas al Proyecto Educativo Institucional (PEI), que señala un camino de realizaciones para el mundo educativo.

Los actuales desafíos que conlleva el sentido de mejora en un centro educativo, pasan por cambiar el paradigma en los procesos de gestión, donde las respuestas creativas e innovadoras a problemáticas antiguas y emergentes hacen la diferencia en resultados de calidad (Gairín, 2004). Para el autor, ya el control y la norma no son las marcas diferenciadoras de gestiones escolares actuales, ya que las demandas sociales actuales les exigen desenvolverse en un medio en continuo cambio y de gran complejidad.

Los retos en la sociedad del conocimiento comienzan a trascender a las políticas micro del centro educativo, donde la gestión para el cambio y mejora escolar pasan a considerarse ejes claves y sustantivos de ahora en adelante, "El cambio educativo depende de lo que el profesor haga y piense: es tan simple y tan complejo como eso" (Fullan, 2002, p. 141).

Una escuela que asume la tarea de mejorar es aquella donde todos sus integrantes plantean sus metas en objetivos comunes, incluidos aquellos roles que muchas veces parecen ser antagónicos, como los de profesores y directivos.

Constituir Comunidades Profesionales de Aprendizaje (CPA), denominadas por Fullan y Hargreaves (1999) como culturas de trabajo cooperativo, debe ser la característica de las escuelas eficaces que han logrado importantes avances en la construcción de ambientes sanos para el desarrollo profesional y el aprendizaje de los alumnos.

La Organización para la Cooperación y el Desarrollo Económicos (OCDE) orienta sobre dónde deben situarse las principales políticas educativas de cara a escuelas más eficaces, y es así que señala como primer gran factor de transformación hacia la mejora educativa las actuaciones de los docentes y en un segundo lugar la gestión y el liderazgo directivo. Por tanto, los nuevos retos de las escuelas serán consolidar liderazgos inclusivos, participativos y pedagógicos (OCDE, 2009).

El Organismo Internacional identificó cuatro áreas de acción claves para que las prácticas de liderazgo escolar produzcan efectos en la mejora de los resultados escolares. Esas áreas destacan la necesidad de (re) definir las responsabilidades del liderazgo escolar, concentrándose en funciones que pueden mejorar los resultados escolares; distribuir el liderazgo escolar, comprometiendo y reconociendo una participación más amplia en los equipos de liderazgo; desarrollar habilidades para un liderazgo escolar eficaz a lo largo de diferentes etapas de la práctica; hacer del liderazgo escolar una profesión más atractiva al asegurar salarios y perspectivas de carrera apropiados.

Este análisis y propuesta de la OCDE (2009) establece la necesidad de develar las reales tareas e interacciones que desarrollan cotidianamente los directores y directoras escolares, ya que permanece en relativa ignorancia la dimensión que puede o no ejercer un líder escolar: "Parece ser, pues, que las decisiones de las personas que desempeñan tareas directivas se toman más por intuición, por sensaciones y corazonadas que como resultado de diagnosis y análisis" (OCDE, 2009, p. 40). 
En este sentido, se reconoce un avance hacia nuevas formas de gestionar la escuela de la mano de liderazgos más democráticos y extendidos y que poco a poco comienzan a recaer en otros actores como los profesores.

El liderazgo pedagógico se entiende como un grupo de gente que aprende y trabaja junta en una Comunidad Profesional de Aprendizaje con un sentido de propósito compartido. Es una escuela vivida como comunidad, los docentes comparten el liderazgo y la toma de decisiones, tienen un sentido compartido de acción, participan en el trabajo colaborativo y aceptan su responsabilidad por los resultados de su trabajo. (Bolívar, 2012, p. 15).

Si existe el reconocimiento del valor transformador del profesor dentro del aula es necesario comenzar a evaluar sus capacidades de liderazgo en relación a la innovación y calidad de los aprendizajes logrados en sus estudiantes (Maureira \& Rojas, 2013).

El constituir Comunidades Profesionales de Aprendizaje (CPA), según Fullan y Hargreaves (1999), será el nuevo camino hacia escuelas más eficaces y que aseguren reales aprendizajes de los estudiantes y de los actores que se comprometen con nuevos roles de colaboración y trabajo profesional.

Cada comunidad educativa deberá adquirir las capacidades suficientes para asumir las distintas tareas que requiere liderar una escuela, es por ello que destaca la importancia de la formación continua que deberán tener los profesores líderes (Antúnez, 2000), incluyendo la reflexión común sobre sus prácticas pedagógicas (Imbernón, 2005).

Las nuevas competencias a desarrollar en los profesores obligan un reconocimiento de su labor profesional en ámbitos de la dirección y gestión del centro educativo, dotándolo de la autoridad suficiente para desempeñar reales funciones de liderazgo (Bolívar, 2012).

Estas condiciones óptimas donde el profesorado pueda desarrollar toda su acción educativa, incluyendo la de un liderazgo pedagógico, serán más factibles de encontrar en centros educativos con un real proyecto de inclusión (Essomba, 2006; Murillo et al., 2010; Sarto \& Venegas, 2009).

La tendencia actual de conceptualizar el liderazgo con características de distribución (Hargreaves \& Fink, 2008) apunta a una nueva forma de entender la escuela que es descrita con las siguientes características (Murillo et al., 2010): cultura de colaboración a partir de un compromiso e implicación efectiva de todos los miembros de una comunidad, de manera tal que el liderazgo esté presente en todos los niveles; que los que detentan el rol de autoridad democraticen las decisiones a partir de prácticas distribuidas, descentralizando el poder; también debe agregarse que el liderazgo distribuido emerja como resultado de un proceso construido en comunidad y que involucra misiones y propósitos compartidos (Bolívar, 2012).

La organización actual de la escuela requiere por tanto que sus líderes transiten de posiciones meramente administrativas a ser agentes de cambio, contando con la complicidad de todos los miembros de la comunidad educativa (Delgado, 2005; González, 2008; Murillo et al., 2010).

Esta distribución del liderazgo no evade de la responsabilidad última a los equipos de dirección de sus funciones inherentes al cargo, ya que "es claro que no hay centro escolar que funcione bien sin un equipo directivo detrás que anima, apoya y potencia — de modo colectivo- las acciones individuales" (Bolívar, 2012, p. 171), pero posibilita y en muchos 
casos faculta para que las decisiones sean tomadas en clave democrática y participativa, lo que favorecería un sentido de mayor comunidad y autonomía que es por lo que luchan muchos centros escolares hoy.

Bajo estas características de liderazgo distribuido situamos a las Comunidades de Aprendizaje (CCA) que se fundamentan en el aprendizaje dialógico que permite una reconstrucción de la escuela y la sociedad (Elboj et al., 2002, p. 38).

El proyecto de Comunidad de Aprendizaje (CdA) se destaca por sobre otros proyectos relacionados con la inclusión educativa, por poseer orientaciones pedagógicas hacia el éxito académico, partiendo del convencimiento que todas las personas tienen capacidades para lograr sus metas; son las instituciones sociales como las escuelas, las que deben prodigarse por no segregar ni acentuar el fracaso escolar con políticas de discriminación de ningún tipo: étnica o cultural, de nivel socio-económico, de discapacidad, etc. (Elboj et al., 2002).

Se proyecta, por tanto, la creación de una cultura educativa equitativa que involucra el gran desafío de re-pensar la educación, el rol de sus actores, las ideas de mundo que se construyen en la cotidianeidad de la escuela y tratar de comprender de mejor forma los fenómenos que esta realidad trae consigo. Es en esta línea que los diversos aportes de Ainscow $(1999,2001,2004)$ y Ainscow y West (2008) aluden a responder preguntas esenciales: ¿cómo crear contextos educativos a los que puedan acceder todos los estudiantes? y ¿de qué manera el sistema educativo deja de excluir a partir del fantasma del fracaso escolar?

Con relación a las CCA existen muchos avances demostrados en diferentes tesis ${ }^{2}$ desarrolladas en los últimos años sobre temas de voluntariado, grupos interactivos, aprendizaje dialógico en relación con la inclusión social, entre otras. Sin embargo, no aparece ninguna en relación con el fenómeno del liderazgo ni las mejores formas de gestionar y liderar la creciente diversidad y complejidad que presentan estos centros educativos.

No basta que una escuela se declare inclusiva y participativa para realmente serlo, es por ello que analizar la cultura escolar por dentro (Rockwell, 1995; Woods, 1985) conforma un proceso de transformación y aprendizaje para todos.

\section{CARACTERÍSTICAS DE LA ESCUELA Y DE LOS PARTICIPANTES}

El Centro de Educación Infantil y Primaria (CEIP) Lledoner de la localidad de Granollers (Cataluña) funciona desde el curso 2005-2006 conformándose desde sus orígenes como una Comunidad de Aprendizaje (CdA). Este centro público atiende a una gran población inmigrante de diversas culturas que dotan a la escuela de una gran heterogeneidad y complejidad.

Nace con un par de cursos de preescolar (Pre-Kinder) y con la idea de impulsar el proyecto de Comunidad de Aprendizaje (CdA), el cual se fundamenta en la participación de familias y voluntarios dentro del proceso educativo de los estudiantes y una idea conjunta de transformación y mejora del contexto social inmediato a la comunidad.

\footnotetext{
Comunidades de aprendizaje: Una práctica educativa de aprendizaje Dialógico para la sociedad de la Información (Valls, 2000); Comunidades de aprendizaje: Un modelo de educación antiracista en la sociedad de la información (Elboj, 2001); Comunidades de aprendizaje: De la segregación a la inclusión (García, 2004); Los grupos interactivos: Una práctica de las comunidades de aprendizaje para la inclusión del alumnado con discapacidad (Molina, 2007); Voluntariado en la escuela: Un estudio de casos dentro del proyecto comunidades de aprendizaje (Viera, 2011); La contribución de las comunidades de aprendizaje al desarrollo de la competencia social y ciudadana (Comte, 2014); Pràctiques Dialògiques a 1’Educació Secundaria (Petreñas, 2014).
} 
La escuela funciona con un equipo directivo integrado por el director, jefa de estudios y secretaria, un claustro de profesores, Personal de Administración y Servicio (PAS) y la Asociación de Madres y Padres (AMPA) como cualquier otra escuela pública española. Sin embargo, en su estructura interna de funcionamiento destacan instancias de participación propias del proyecto de $\mathrm{CdA}$.

La escuela se organiza en comunidades: pequeños, medianos y mayores. Cada comunidad cuenta con un representante de los profesores que se integra, junto al equipo directivo, en lo que se denomina equipo de coordinación o equipo de liderazgo ampliado, que es el responsable de dirigir, gestionar y tomar decisiones que en otras escuelas solo serían atribución del equipo directivo o incluso únicamente del director.

Existe dentro de la escuela un enorme sentido de pertenencia al proyecto educativo que ellos libremente han decidido soñar y construir, y para lograr sus metas necesitan cambiar ciertos funcionamientos y estructuras internas de la organización. Es así como se puede distinguir una organización descentralizada del poder, basada en procesos de gestión democráticos y participativos que permitirán que muchos actores puedan asumir roles protagónicos de liderazgo. Esta estructura horizontal de gobierno tiene por misión brindar el máximo apoyo a las actividades pedagógicas y extraescolares de la escuela para lograr mejores aprendizajes de todos los miembros de la comunidad (Viera, 2011).

La estrecha vinculación entre familia-escuela y entorno crea un modelo de escuela abierta con procesos deliberativos en la toma de decisiones y permite que existan relaciones interpersonales igualitarias basadas en los mejores argumentos. Esta filosofía es denominada por sus actores y comunidad científica internacional como aprendizaje dialógico (Valls, 2000), el cual fomenta una participación directa y de corresponsabilidad en los aprendizajes de los estudiantes en primer lugar, y luego en los propios miembros de la escuela que colaboran y se enriquecen de la experiencia de educar.

Las distintas voces que se dan cita dentro de esta comunidad de aprendizaje son validadas por todos los actores, y es así que estudiantes, profesores, padres, madres, personal administrativo y de servicio y voluntarios externos tienen derecho a expresarse en la misma medida que el equipo de dirección. La participación y colaboración se transforman así en la manera de articular la comunicación y el diálogo, sea en función de construir acuerdos en cuanto a la mejora como también para resolver problemas de la forma más expedita.

La manera ejecutiva de llevar a la práctica estas orientaciones comunicativas y participativas se logra a partir de la creación de comisiones mixtas de trabajo (10 en total), donde se distribuyen las múltiples funciones sociales, económicas, formativas y administrativas que requiera gestionar la escuela. Cada comisión mixta es integrada por profesores, apoderados y voluntarios, y se organizan en base a un plan de gestión general que marca la comisión gestora, que es la que toma los acuerdos más generales de funcionamiento en la escuela y que posee la característica de ser triestamental.

En esta comisión mixta participan representantes de los apoderados y familias voluntarias, de los profesores y del equipo dirección, es la máxima instancia para la toma de decisiones, muy parecido a lo que son los consejos escolares chilenos (Ley 19.979, Decreto 24/2005), pero con mayores atribuciones y autonomía pedagógica y financiera.

Otro de los aspectos importantes de consignar de la comunidad de aprendizaje estudiada se refiere a las metodologías de enseñanza y aprendizaje innovadoras que presenta, y que junto a la estructura de organización participativa constituyen los principales fundamentos de cambio y mejora educativa que aporta este tipo de proyectos de transformación. Estas 
metodologías innovadoras e inclusivas están pensadas para grupos heterogéneos de estudiantes, donde no se discrimine por el mayor o menor conocimiento que posean ni por los capitales culturales de sus familias ni menos por su lugar de procedencia ni credo ni idioma.

Bajo esta perspectiva de máxima inclusión y aceptación de la diversidad es que todas y todos son llamados a colaborar en el aprendizaje de los estudiantes, creándose espacios formales destinados a aquello. Son más de 90 personas las que actúan como voluntariado permanente en la escuela, cifra que aumenta en celebraciones emblemáticas (carnavales, fiestas comarcales y fiestas de fin de curso).

El primero de esos espacios de inclusión pedagógica son los Grupos Interactivos (GI), en los que el profesor de cada curso debe liderar y convocar a las familias y voluntarios a diseñar e implementar pequeñas guías de aprendizaje con los estudiantes. Estas guías se ejecutan en materias troncales como Matemáticas y Lenguaje, y dentro del horario normal de clases. Estos GI son reconocidos como una de las mayores innovaciones tendientes a la inclusión de grupos desfavorecidos, como estudiantes con discapacidades (Molina, 2007)

La manera de hacerlo es bastante simple, ya que el profesor y los voluntarios-familias crean al comienzo de un curso una serie de guías de trabajo que luego implementan en pequeños grupos de estudiantes. La idea es que dentro del tiempo de una clase existan cuatro grupos de trabajo diferentes dirigidos por un padre o madre y coordinado por el profesor, y que los estudiantes roten cada cierto periodo de tiempo, logrando que se rompa aquella tendencia donde el profesor educa aisladamente en su sala de clases, por el contrario se potencia que exista una real participación pedagógica de los padres y madres.

La otra actividad de inclusión es denominada Tertulias Literarias (TL), y consisten en la lectura compartida y dialogada entre padres y madres y los estudiantes de un curso que, por lo general, no es el curso de sus propios hijos, sino que puede ser cualquier otro, ya que lo importante es lograr aprendizajes de calidad para toda la escuela.

Estas herramientas de trabajo intergeneracional e intercultural dotan al centro escolar de una gran riqueza. A ellas se les suman el trabajo por proyectos educativos y el aprendizaje por rincones de conocimientos.

Estas últimas innovaciones rompen el currículum de contenidos establecidos en la lógica de buscar aprendizajes profundos y de real significado para los estudiantes, así como también favorecer el trabajo colaborativo entre los colegas y las familias. La consecuencia lógica de estas metodologías y estructura organizativa democrática es un alto grado de satisfacción laboral y grato ambiente educativo para todos los miembros de la escuela. Muchos de ellos declaran la gran demanda de trabajo, tiempo y compromiso que involucra este tipo de organización, pero que vale la pena por los logros educativos que ha tenido la escuela y la satisfacción de ver a los niños y niñas venir felices a estudiar, y a padres agradados en compartir y colaborar.

Como hemos podido apreciar en el caso de esta escuela, el factor de liderazgo se relaciona directamente con una gestión de la escuela inclusiva, abierta a todos los que quieran comprometerse y en constante innovación hacia la mejora de los aprendizajes de los estudiantes.

En la actualidad la escuela cuenta con más de una treintena de profesionales que imparten docencia en 18 cursos, con la expectativa de consolidar los logros de estos primeros ${ }^{3}$ años,

En el curso 2013-2014 se produce la graduación de la primera generación de estudiantes y familias que dieron inicio al proyecto de CdA. 
siguiendo la ruta trazada en la consecución de sus objetivos surgidos de la fase de "sueño": una fase inicial del proyecto CdA que consiste en recoger y organizar colectivamente los sueños que, en relación al futuro de la escuela, han trabajado separadamente el alumnado, el profesorado y las familias.

\section{ENFOQUE Y METODOLOGÍA DE INVESTIGACIÓN}

Siguiendo nuestro objetivo general de investigación: Comprender los significados y alcances del fenómeno del liderazgo y sus repercusiones en la mejora del proyecto educativo de comunidad de aprendizaje, optamos por una metodología de corte cualitativo. La importancia de conocer la escuela por dentro (Rockwell, 1995; Woods, 1985) su funcionamiento no develado, las interacciones y subjetividades que se dan entre los actores, se transforma en una necesidad para analizar en el ámbito del liderazgo.

Se optó por un estudio de casos (Stake, 1999), ya que buscábamos profundizar en la construcción de significados y percepciones a partir de dar respuesta a las siguientes interrogantes: ¿Cómo se construye el liderazgo en el contexto del proyecto de Comunidades de Aprendizaje? ¿Qué percepciones y significados le atribuyen el PAS, profesores, familias voluntarias y los miembros del equipo directivo? y ¿Qué valoración hacen del papel del liderazgo en su propia comunidad?

Para la obtención de datos se utilizaron diversos instrumentos que asegurasen un mayor rigor científico, junto con los aportes del paradigma comunicativo-crítico (Gómez et al., 2006), estableciendo un diálogo igualitario con los protagonistas de la CdA que enriqueciera y proporcionara validez a las evidencias obtenidas a través de la observación y de la exploración documental. Concretamente, se utilizaron cuatro instrumentos para recoger información: observación participante, revisión de documentos internos, entrevistas en profundidad y grupos de discusión (ver Tabla 1).

Tabla 1. Instrumentos de recolección de información

\begin{tabular}{|l|l|}
\hline \multicolumn{1}{|c|}{ Instrumentos } & \multicolumn{1}{|c|}{ Informantes } \\
\hline $1^{\circ}$ Observación participante & Visitas reiteradas al centro escolar. Uso de diario de campo. \\
\hline $2^{\circ}$ Revisión de documentos internos & $\begin{array}{l}\text { Proyecto de Autonomía de Centro (PAC), Proyecto de } \\
\text { Dirección del Director aprobado hasta 2014. Plan Anual de } \\
\text { Trabajo (2012-13), Actas de reuniones de Claustro. }\end{array}$ \\
\hline $3^{\circ}$ Entrevistas en profundidad & $\begin{array}{l}5 \text { Entrevistas a los miembros del equipo directivo/ } \\
\text { Entrevistas a familias no voluntarias/3 Entrevistas a familias } \\
\text { voluntarias de la AMPA/ 3 Entrevistas a Profesores de la } \\
\text { escuela/ 2 Entrevistas al PAS. }\end{array}$ \\
\hline $4^{\circ}$ Grupos de discusión & $\begin{array}{l}1 \text { Grupo de discusión de profesores/ 1 Grupo de discusión de } \\
\text { familias-voluntarios. }\end{array}$ \\
\hline
\end{tabular}


Estudios Pedagógicos XLIII, $\mathrm{N}^{\circ}$ 1: 41-59, 2017

EL LIDERAZGO COMPARTIDO COMO FACTOR DE SOSTENIBILIDAD DEL PROYECTO DE COMUNIDADES DE APRENDIZAJES

En la tabla siguiente (ver Tabla 2) se esquematizan los principales criterios de rigor científicos, siguiendo la propuesta de Lincoln y Guba (1985, adaptada desde Vasilachis, 2009).

Tabla 2. Resumen de procedimientos de rigor científico empleados en la investigación

\begin{tabular}{|l|l|}
\hline Metodología Cualitativa & \multicolumn{1}{|c|}{ Procedimientos empleados en el estudio de casos } \\
\hline Credibilidad & $\begin{array}{l}\text { Observación y permanencia en el campo de estudio como voluntario e } \\
\text { investigador durante tres cursos académicos/ Triangulación de las } \\
\text { fuentes de datos (expertos, metodológica, interesados)/ Validación } \\
\text { comunicativa. }\end{array}$ \\
\hline Dependencia & $\begin{array}{l}\text { Utilización de una planificación a partir de un diagrama de flujo/ } \\
\text { Establecimiento de pistas de revisión dentro del informe final de tesis/ } \\
\text { Auditoría de dependencia al contar con dos directores de tesis } \\
\text { especialistas en sus respectivas áreas. }\end{array}$ \\
\hline Confirmabilidad & $\begin{array}{l}\text { Ejercicio contante de reflexión para captar la realidad desde la } \\
\text { subjetividad de los actores participantes: significados, percepciones, } \\
\text { vivencias y experiencias. }\end{array}$ \\
\hline Transferibilidad & $\begin{array}{l}\text { Descripciones densas y precisas del contexto y de los participantes del } \\
\text { estudio de casos/ Recogida de abundante información proporcionada } \\
\text { desde los participantes e instrumentos utilizados. }\end{array}$ \\
\hline
\end{tabular}

Nota: propuesta de Lincoln y Guba (1985) adaptada desde Vasilachis (2009).

\section{PROCESO DE ANÁLISIS DE LOS DATOS}

Con los instrumentos anteriores se obtuvo una gran cantidad de información en bruto. Para su reducción y para facilitar la emergencia de datos cualitativos más organizados utilizamos el programa de análisis cualitativo ATLAS TI versión 5.3. Una vez revisados todos los documentos ingresados (entrevistas y grupos de discusión) procedimos a generar una lista de recurrencias con los temas que se destacaban en los fragmentos seleccionados, llegando a confeccionar una primera codificación tentativa que sería analizada por los investigadores para verificar que al comparar las diversas categorías por su descripción no se solaparan unas con otras y que estas primeras categorías permitieran un desarrollo sucesivo posterior en cuanto a seguir avanzando de lo más superficial a lo más profundo y de lo más amplio a lo más concreto.

Preliminarmente construimos un sistema de 20 categorías distribuidas en 5 metacategorías de forma inductiva, las cuales ingresamos a la Unidad Hermenéutica (HU) a través de la creación de códigos libres (free codes). Una vez creadas estas 20 categorías en la HU se procedió a seleccionar los fragmentos ya creados y asociarlos a los códigos pertinentes de la lista (code by list). Esta fase nuevamente nos tomó su tiempo, ya que requería un mayor análisis para que las unidades de significado (US) seleccionadas fueran representativas para cada uno de los códigos elaborados. En algunos casos fue 
preciso eliminar algunas US que previamente parecían interesantes o relevantes, pero que posteriormente no se sustentaban.

Paralelamente creamos nuevos comentarios (memo), esta vez asociándolos a los códigos (codes). Una vez ingresados todos los fragmentos con sus respectivos códigos pudimos establecer sub-categorías y definir cada uno de los códigos desde el code manager, con la ayuda de los memos asociados. Finalmente, llegamos a la creación de diversos informes (out puts) considerados los primeros reportes de análisis de nuestros principales instrumentos de recolección de evidencias de campo, como fueron las entrevistas y los grupos de discusión.

El segundo momento del análisis consistió en re-pensar estas primeras categorías surgidas con la colaboración del programa informático Atlas ti, para llegar a la reelaboración de las mismas. Esta vez el trabajo fue tomar los reportes de códigos y lista de fragmentos (codes-quotations list) y comenzar a desmenuzarlos por el método de comparación constante, es decir, comparar cada fragmento con la categoría asignada y con fragmentos similares, repensando, sacando, poniendo, agrupando etiquetas hasta llegar a la elaboración final de las 3 Meta-categorías, 9 categorías y 29 subcategorías.

El paso final que otorgaría mayor credibilidad a nuestro sistema categorial fue la definición conceptual de cada una de las categorías que a continuación presentamos y que son una parte fundamental para el análisis e interpretación a las que hemos arribado (ver Figura 1).

Figura 1. Mapa relacional entre las categorías del estudio

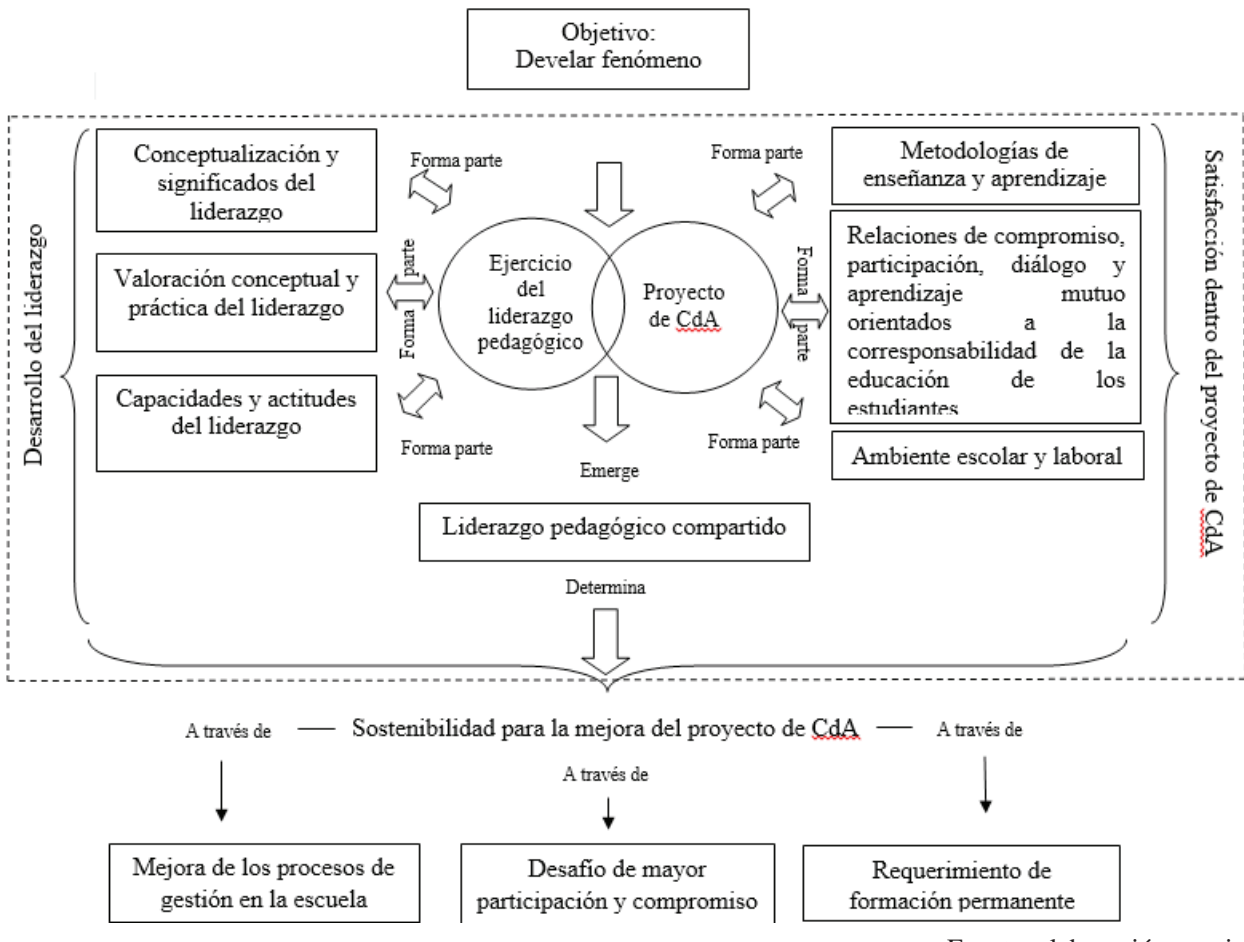

Fuente: elaboración propia. 
En este esquema conceptual, las categorías que directamente se relacionan con el ejercicio del liderazgo pedagógico son 3, agrupadas dentro de la metacategoría Desarrollo del liderazgo. Por su parte, las categorías que se desprenden de la evaluación que hacen los actores del proyecto de CdA también son 3, agrupadas dentro de la metacategoría Satisfacción dentro del proyecto de CdA. Lo que resulta de interés en nuestra investigación es que el cruce de dichas categorías hace emerger con fuerza el concepto de Liderazgo Pedagógico Compartido como un elemento que distingue a la institución y que configura una metacategoría que denominamos Sostenibilidad para la mejora del proyecto de CdA. En ella se establecen los desafíos y requerimientos de mejora que la escuela declara urgentes para consolidar el proyecto educativo que defienden y sueñan. Sustentados en esta constatación, pasamos a dar cuenta de los principales resultados que dan respuesta a nuestras preguntas de investigación y objetivos del estudio.

\section{DISCUSIÓN DE LOS RESULTADOS DE LA INVESTIGACIÓN}

\subsection{TIPOS DE LIDERAZGO PRESENTES EN LA INVESTIGACIÓN}

\subsubsection{Liderazgo pedagógico compartido}

El ejercicio del liderazgo pedagógico dentro de esta CdA es entendido como aquel que busca la mejora de los aprendizajes de los estudiantes, estableciendo la responsabilidad en ello de los profesores, los miembros del equipo directivo y las familias y voluntariado del centro. Es así que los actores prefieren conceptualizar el liderazgo escolar que se desarrolla al interior de la CdA como un liderazgo compartido.

No hay una persona sola que dinamice... el equipo directivo conjuntamente con el equipo de coordinación dinamizan el equipo de maestros que forman las diferentes comunidades pequeños, medianos, mayores. También, las comisiones mixtas. Para mí sin ellas la escuela estaría a la mitad de la mitad de la mitad de lo que está. (P14: Entrevista Secretaria del Equipo directivo.14:7).

\subsubsection{Liderazgo docente}

El liderazgo de los profesores se establece en tres vertientes: una en relación con la responsabilidad profesional frente a la institución escolar, caracterizada por seguir las directrices que les marca el proyecto educativo de $\mathrm{CdA}$ y por el reconocimiento y auto-reconocimiento de ese liderazgo, "Yo personalmente como profesor dentro de mi pequeña parcela soy líder en este sentido, guío bajo unas directrices que son los ítems claros de la comunidad de aprendizaje e intento guiar de la mejor manera posible" (P13: Entrevista Profesor 3. 13:45).

Una segunda vertiente se vincula al acompañamiento de colegas nuevos, para conseguir una relación de colaboración entre veteranos y novatos, dirigida al conocimiento del funcionamiento de la escuela como CdA.

Sobre todo profesores, los que somos tutores, bueno ahora me pongo como tutora, yo creo que sí, porque nos ven como compañeros, no nos ven como líderes ni mucho 
menos. Yo no me considero líder, pero con otros compañeros que también son los que ya llevan más tiempo y son más veteranos y pueden verse como un modelaje, con la capacidad de escuchar, de acompañar, de acompañar el primer llanto de los primeros días del Lledoner. (P12: Entrevista Profesor 2. 12:55).

En tercer lugar debemos considerar el liderazgo del profesor frente a los estudiantes y su trabajo en el aula, situación reconocida como el espacio normal del desarrollo profesional de los docentes. Se destacan atributos como la importancia de ser un líder positivo frente a los estudiantes y el trabajo de coordinación que realiza el profesorado con las familias voluntarias que les colaboran dentro del aula:

A nivel de profesores encuentro que es un tema muy difícil y que muchas veces no es consciente del "poder" que pueda tener un maestro. De que en realidad los niños acaban copiando todo aquello que ven, sobre todo si viene de un adulto y de la importancia de crear un vínculo para que el liderazgo no se convierta en algo impuesto, sino que sea algo natural y positivo; también para los niños a la vez. (P11: Entrevista Profesor 1. 11:3).

\subsubsection{Liderazgo participativo de las familias voluntarias}

El liderazgo de las familias voluntarias es uno de los elementos claves a la hora de hablar del proyecto de $\mathrm{CdA}$, y en el caso investigado es reconocido como trascendente por el impacto que provoca en distintas esferas de la escuela. Es así que tanto el equipo directivo como los profesores reconocen la existencia de un liderazgo participativo de madres y padres que se comprometen con la educación de todos los niños y niñas de la escuela, asumiendo diversas tareas y responsabilidades sin que nadie los obligue.

La suerte que hemos tenido es que varias familias también han liderado muchísimo y si vas aquí en las comisiones tenemos vocales y muchos de estos vocales lideran, asumen responsabilidades y lideran y tienen una visión muy buena y muy clara de hacia dónde queremos ir, tanto en lo pedagógico como en cualquiera de los ámbitos que gestiona cada comisión. (P 3: Entrevista Director 1. 3:36).

Las familias voluntarias que participan activamente dentro de las comisiones y de la AMPA se reconocen como representantes y colaboradores más que como líderes:

Nosotros los que estamos representando al AMPA se nos conoce porque tenemos el cargo y vamos a más reuniones que el resto y estamos en más sitios, pero no creo, no entiendo que haya mucho liderazgo en todo el colegio, no determinante por lo menos. Hay pequeños grupos y luego lo que se hace es juntarnos todos, todos los vocales, pero es bastante democrático [ríe] hasta el momento. (P16: Entrevista Voluntario 2. 16:9).

Esta situación es observada no como un contrasentido sobre el rol de liderazgo que puedan tener o no las familias, sino más bien es significada como una actitud de respeto y deferencia hacia el claustro de profesores que en la misma sintonía sí reconoce la gran labor que realizan las familias dinamizando el centro educativo. 
Siempre decimos que hay pocos voluntarios o que hay gente que no se implica o lo que sea, pero cuando tu sumas todos los voluntarios que tenemos desde los tres años hasta sexto son muchos, es impresionante, o sea porque todos hacemos grupos interactivos, hacemos tertulias, sí que algún curso a veces cuesta más, pero bueno, si tú vas sumando un centenar tendremos y esto en una escuela a mí me parece espectacular, es mucha gente. (P18: Grupo de discusión de profesores. 18:19).

\subsubsection{Otras visiones de liderazgo}

Encontramos cinco agrupamientos de significados distintos a los anteriormente presentados, que emergen de las evidencias y que complementan aquella idea de la polisemia sobre el concepto de liderazgo.

El primero de ellos lo calificamos como "caracteres y capacidades innatas de las personas", el segundo grupo de significados fue "negativa a aceptar la idea de ser líder", el tercer agrupamiento "concepto de liderazgo difuso", un cuarto grupo lo denominamos "cuando te etiquetan de líder" y, finalmente, el último grupo llamado "liderazgo de estudiantes".

\subsubsection{Valoración del liderazgo}

Existe una valoración positiva del ejercicio del liderazgo cuando ha colaborado para que los propios actores comiencen un proceso reflexivo en relación al camino recorrido en función de los objetivos o sueños aún por cumplir, y cuando sirve para fortalecer el proyecto de escuela de CdA con el aporte de todos.

Si los aspectos claves del liderazgo se asumen, pues la comunidad sale muy fortalecida y si no, pues la comunidad por muy buen proyecto que sea, por muy coherente que sea, incluso aunque haya un buen nivel de participación de base, pues la comunidad se debilita, el funcionamiento se debilita y eso lo hemos vivido en el colegio desde el principio. (P 3: Entrevista Director 1. 3:64).

Una parte minoritaria del profesorado entiende el concepto de liderazgo como mando autoritario, lo cual provoca una situación que evidencia cierto malestar ante actuaciones puntuales de algunos de sus líderes.

Cuando encuentro a faltar liderazgo es cuando se torna muy exagerado, impuesto, demasiado autoritario entonces sí que te das cuenta que está, a veces cuando es el adecuado, queda difuminado. La gente no acaba de ser consciente cuando tendría que ser algo que se tuviese que potenciar, encuentro que sí falta liderazgo positivo. (P11: Entrevista Profesor 1. 11:4).

\subsection{CARACTERÍSTICAS Y PERFIL DEL LIDERAZGO PEDAGÓGICO COMPARTIDO}

A partir de todas las voces que nos colaboraron en la investigación fue posible construir una tabla con un perfil de capacidades y actitudes de tipo comunitario ideales de desarrollar al interior de la escuela. Cabe mencionar que este agrupamiento fue construido desde el re- 
conocimiento que hacen los propios actores sobre características (acciones y aptitudes) que tienen algunos se sus líderes, que en la mayoría de los casos corresponde a roles de director, ex secretario del equipo directivo, vicepresidente de la AMPA, jefa de estudios y profesores de mayor veteranía. Estas capacidades y actitudes llegan a favorecer el buen ambiente de trabajo y de aprendizaje que desarrolla en la escuela y que es destacado por muchos de sus actores como un factor de satisfacción para seguir colaborando (ver Tabla 3).

Tabla 3. Perfil del liderazgo pedagógico compartido en la CdA

\begin{tabular}{|l|l|}
\hline \multicolumn{1}{|c|}{ Capacidades y habilidades } & \multicolumn{1}{|c|}{ Valores y actitudes } \\
\hline $\begin{array}{l}\text { Capacidad de escucha, diálogo y comunicación: } \\
\text { argumentación, influir en otros, llegar a } \\
\text { consensos, empatía. }\end{array}$ & $\begin{array}{l}\text { Compromiso y automotivación: implicación } \\
\text { con el proyecto de CdA. }\end{array}$ \\
\hline $\begin{array}{l}\text { Capacidad de trabajo en grupos: animar y tirar } \\
\text { del carro, incentivar, impulsar. }\end{array}$ & $\begin{array}{l}\text { Optimismo: Actitud positiva frente a los } \\
\text { problemas. }\end{array}$ \\
\hline $\begin{array}{l}\text { Capacidad reflexiva: autocrítica, inteligencia, } \\
\text { paciencia, control de estrés, equilibrio emocional. }\end{array}$ & Alegría: contagiar entusiasmo, buen carácter. \\
\hline $\begin{array}{l}\text { Capacidad creativa: agilidad mental para la } \\
\text { resolución de conflictos, proponer mejoras, } \\
\text { emprender con acciones innovadoras. }\end{array}$ & $\begin{array}{l}\text { Respeto y tolerancia: a las opiniones de los } \\
\text { otros, a las decisiones unilaterales, a la } \\
\text { experiencia y veteranía. }\end{array}$ \\
\hline $\begin{array}{l}\text { Capacidad organizativa: exigencia por el trabajo } \\
\text { bien hecho, tomar decisiones, gestión de recursos } \\
\text { humanos y económicos. }\end{array}$ & Solidaridad: acompañamiento y ayuda. \\
\hline
\end{tabular}

Fuente: elaboración propia.

Valores como la tolerancia, el respeto, la solidaridad, alegría, optimismo, compromiso y la automotivación son vivenciados en esta escuela, pasando a constituirse como esenciales en la construcción de un perfil integral de liderazgo y que aporta directamente a la sostenibilidad del proyecto de escuela.

A su vez, las capacidades de diálogo, escucha y comunicación se establecen como articuladoras de procesos de gestión, trabajo en equipo y resolución de conflictos que para una organización educativa pueden ser la clave del éxito o el fracaso, según cómo sean administradas y lideradas.

\subsection{SATISFACCIÓN Y SOSTENIBILIDAD DEL PROYECTO DE CDA}

Sin duda, la máxima valoración positiva que pudimos extraer sobre el proyecto interno de escuela se encuentra en las relaciones que se establecen entre los diferentes actores de la CdA. Esto es destacado por el claustro de profesores, equipo directivo, personal de administración y servicio y representantes de la AMPA, familias y voluntarios. 
Estas relaciones se establecen en términos de diálogo, aprendizaje mutuo, compromiso, participación y corresponsabilidad en la educación de los estudiantes entre estos mismos actores que declaran sentirse satisfechos por el nivel de buenas relaciones humanas logradas, que se trasunta en ambientes laborales óptimos para desempeñarse y una sensación de bienestar no muy común en la mayoría de centros educativos, pero que en esta CdA se destaca como una de sus grandes fortalezas.

La participación y el compromiso de las familias voluntarias son esenciales para dotar de sostenibilidad al proyecto interno de la escuela y surgen como un elemento de satisfacción reconocido por todos los miembros de la comunidad educativa, "No podríamos ser comunidad de aprendizaje si no estuvieran las familias, y si este pedazo de proyecto y pedazo de escuela tuviera que funcionar solamente por el equipo de maestros esto no funcionaría." (P18: Grupo de discusión de profesores. 18.124)

Otro de los elementos de satisfacción está dado por la implementación de metodologías de enseñanza y aprendizaje consideradas innovadoras, creativas e inclusivas que se transforman en la principal motivación de los actores para permanecer dentro de la escuela. Tanto profesores, equipo directivo y en especial las familias-voluntarias destacan esta fortaleza: "Piden mucho que los niños se comuniquen y se respeten, además tienen las tertulias y los proyectos de participación con las familias y el trabajo en pequeños grupos con los niños es muy bueno porque pueden estar más por un aprendizaje directo con atención individualizada y enriquecedora.” (P15: Entrevista Voluntario 1. 15:23).

Destaquemos, finalmente, y no por orden de importancia, el carácter inclusivo de la escuela que llama la atención a muchos profesionales, una política que se traduce en no retirar estudiantes con Necesidades Educativas Especiales (NEE) del aula. Se trata de un rasgo diferenciador frente a otras escuelas que sí recurren a prácticas de este tipo, segregando a estudiantes en salas especiales. La escuela destaca este hecho como un acto de inclusión.

Ello da lugar a una relación intensa entre los profesionales en el establecimiento, que se percibe como una oportunidad de trabajo colaborativo y a la vez como un gran desafío de coordinación de las diferentes actividades a desarrollar. Esta política inclusiva es motivo, a su vez, de que muchos docentes quieran trabajar bajo este régimen. "¿Qué es lo que me motiva a permanecer? [...] es la primera vez que no se sacan los niños con dificultades del aula ¡nunca!, entonces justamente esto hace que tengas que tener un trabajo de coordinación con el tutor mucho más intensa y hay que sacar horas de donde sea" (P11: Entrevista Profesor 1. 11:50).

6.4. ELEMENTOS A MEJORAR PARA ASEGURAR LA SOSTENIBILIDAD DEL PROYECTO DE ESCUELA

Los elementos que pueden mermar la sostenibilidad del centro los encontramos principalmente a nivel de gestión y se pueden apreciar en los siguientes elementos. La gestión de la comunicación es fundamental para sostener el proyecto de comunidad. Es destacada por los integrantes del equipo directivo como eslabón que permite el diálogo entre los diversos grupos que colaboran en la organización y dinamización de la escuela, como las comisiones mixtas, equipo directivo, AMPA, profesores, encargados de áreas, comisión gestora.

La gestión externa del centro escolar presenta un estancamiento y una difícil relación con la Administración Educativa. Este es un problema que urge resolver a los miembros de 
la CdA por el beneficio mutuo. Es inviable la sostenibilidad de una escuela que no cuenta con este nivel de respaldo de sus autoridades locales, en especial en la entrega de recursos y en la rendición de cuentas en base a resultados medibles.

Por otra parte, la situación de crisis social y económica que afecta a la totalidad del estado español en los últimos años ha repercutido al interior de la escuela, afectando con sus recortes educativos a las familias, estudiantes y profesores. Esta situación debe ser bien gestionada para evitar nuevas exclusiones y avanzar con la idea de cambio y mejora social que proyecta la escuela a partir de la materialización de sus sueños de comunidad de aprendizaje.

Finalmente, encontramos el componente formativo como otra de las preocupaciones que debe saber gestionar la escuela. Hemos detectado que las actuaciones formativas en relación al liderazgo son escasas y se centran en el equipo directivo y en las personas que detentan algún rol de liderazgo, situación que pugna con la idea de un liderazgo compartido y que en definitiva puede erosionar las bases democráticas desde donde se ha formulado el proyecto educativo.

\section{CONCLUSIONES}

Develar el significado del liderazgo en esta comunidad ha propiciado que sus propios actores iniciaran un proceso reflexivo encaminado a valorar el camino recorrido en función de los objetivos y sueños aún por cumplir. Este diálogo y deliberación constante en un colectivo reflexivo profesional (Imbernón, 2005) configura el primer indicio de cambio hacia la mejora y la transformación de la escuela más allá de la escolarización y funciones administrativas tradicionales.

Como nos muestra este caso de innovación educativa, la idea de establecer un liderazgo compartido en la escuela deberá incluir a todos los miembros (profesores, padres y madres, personal de apoyo y servicio, voluntarios, autoridades locales y directivos) bajo nuevas lógicas de administración de los recursos materiales, humanos y de conocimiento, ya que los objetivos de la institución se orientan hacia los aprendizajes de todas y todos.

Otro de los aspectos importantes sobre el que reflexionar es que el rol de autoridad de los equipos directivos se mantiene en contextos de alta participación y no es delegable en cuanto a responsabilidades, según las voces de las familias y voluntarios. Por lo tanto es posible una democratización de la escuela bajo diversos liderazgos, incluyendo el del equipo directivo que deberá encabezar procesos de empoderamiento de otros agentes que participan en mayor o menor medida por el bien común de la escuela.

Dentro de los agentes que necesitan un mayor poder y liderazgo en los procesos de gestión y administración de las escuelas se encuentran curiosamente los profesores, sin que ello contravenga su labor esencial como líderes pedagógicos dentro del aula. Este empoderamiento convendría encauzarlo hacia procesos de acompañamiento o mentorías de profesores nóveles o principiantes o que ingresan a centros educativos que requieran una inducción por ciertas complejidades que encierran determinados contextos políticos, sociales, culturales e ideológicos.

Finalmente creemos que este tipo de proyectos inclusivos que favorecen la equidad y la justicia social requieren de un gran esfuerzo y compromiso de parte de todos sus miembros, y por tanto necesitan de una constante socialización y tiempo de trabajo que en algunos 
casos puede desgastar ciertos procesos. Por tanto su sostenibilidad dependerá de diversos factores internos (gestión, liderazgo, compromiso profesional, corresponsabilidad en los aprendizajes, participación activa de padres y madres, buen ambiente escolar, metodologías activas y creativas, formación permanente e integración de los recién llegados) como también de factores externos (apoyo de las administraciones educativas, mayores recursos económicos tendientes a la autonomía institucional, contexto social favorable y redes de colaboración e investigación en comunidades de aprendizaje).

Las preguntas que nos surgen a continuación son ¿Qué tan preparadas están las escuelas para asumir el reto de cambiar y transformarse en espacios mucho más democráticos y colaborativos? ¿Es posible un liderazgo pedagógico auténtico de las familias que se implican plenamente en una CdA? ¿Está asegurada la sostenibilidad de proyectos educativos que demandan tiempo y esfuerzo extra a sus integrantes, y que por otra parte deben rendir cuentas a administraciones educativas que desconocen sus particularidades? y ¿Qué nuevos valores emergerán de esta coyuntura? El liderazgo compartido e inclusivo puede llegar a resolver algunas de estas interrogantes, para ello será necesario replantear algunos de los roles históricos de participación de algunos de sus miembros, en especial la de los profesores, estudiantes y familias.

La experiencia descrita relata un momento, un contexto real y posible que no cierra los ojos a nuevas formas de cambio y mejora, quizás allí radique el mayor aprendizaje que deba hacer suyo la institución escuela, que acostumbra a reproducir patrones de éxito sin reflexionar-se y auto-conocerse.

\section{REFERENCIAS BIBLIOGRÁFICAS}

Ainscow, M. (1999). Understanding the Development of Inclusive Schools. Londres: Falmer.

Ainscow, M. (2001). Desarrollo de Escuelas Inclusivas. Ideas, propuestas y experiencias para mejorar las instituciones escolares. Madrid: Narcea.

Ainscow, M. (2004). Developing inclusive education systems: what are the levers for change? Recuperado de http://web.uam.es/personal_pdi/stmaria/sarrio/D-OCUMENTOS, \%20 ARTICULOS,\%20PONENECIAS,/Developing\%20educational\%20inclusive\%20setings

Ainscow, M., \& West, M. (2008). Mejorar las escuelas urbanas: Liderazgo y colaboración. Madrid: Narcea.

Antúnez, S. (2000). La acción directiva en las instituciones escolares: Análisis y propuestas. Barcelona: ICE, Universitat de Barcelona.

Bolívar, A. (2012). Políticas actuales de mejora y liderazgo educativo. Málaga: Aljive.

Comte, C. (2014). La contribución de las comunidades de aprendizaje al desarrollo de la competencia social y ciudadana. Fondo de Tesis Universitat de Barcelona. Recuperado de http://www. tesisenred.net/handle/10803/134877

Delgado, M. (2005). El liderazgo en las organizaciones educativas: Revisión y perspectivas actuales. Revista Española de Pedagogía, 63(232), 367-388.

Elboj, C. (2001). Comunidades de aprendizaje: Un modelo de educación antiracista en la sociedad de la información. Fondo de Tesis Universidad de Barcelona.

Elboj, C., Puigdellivol, I., Soler, M., \& Valls, C. (2002). Comunidades de aprendizaje. Transformar la educación. Barcelona: GRAÓ.

Essomba, M. (2006). Liderar escuelas interculturales e inclusivas: Equipos directivos y profesorado ante la diversidad cultural y la inmigración. Barcelona: GRAÓ.

Fullan, M. (2002). Los nuevos significados del cambio en la educación. Barcelona: Octaedro. 
Fullan, M., \& Hargreaves, A. (1999). La escuela que queremos. Los objetivos por los cuales vale la pena luchar. Buenos Aires: Amorrortu.

Gairín, J. (2004). Mejorar la sociedad, mejorando las organizaciones educativas. En A. Villa (Ed.), Dirección para la innovación: apertura de los centros a la sociedad del conocimiento (pp. 77127). Bilbao: ICE de la Universidad de Deusto.

García, C. (2004). Comunidades de aprendizaje: De la segregación a la inclusión. Fondo de Tesis Universitat de Barcelona.

Gómez, J., Latorre, A., Sánchez, M., \& Flecha, R. (2006). Metodología Comunicativa Crítica. Barcelona: El Roure.

González, M. T. (2008). Diversidad e Inclusión Educativa: Algunas Reflexiones sobre el Liderazgo en el Centro Escolar. Revista Electrónica Iberoamericana sobre Calidad, Eficacia y Cambio en Educación, 6(2), 82-99.

Hargreaves, A., \& Fink, D. (2006). Estrategias de cambio y mejora en educación caracterizadas por su relevancia, difusión y continuidad en el tiempo. Revista de Educación, 339, 43-58.

Hargreaves, A., \& Fink, D. (2008). El Liderazgo sostenible: siete principios para el liderazgo en centros educativos innovadores. Madrid: Morata.

Imbernón, F. (2005). Vivencias de maestros y maestras: compartir desde la práctica educativa. Barcelona: GRAÓ.

Maureira, O., \& Rojas, A. (2013). Características del liderazgo transformacional en los docentes de lenguaje y comunicación en una muestra de establecimientos escolares de vulnerabilidad social. Estudios Pedagógicos, 39(1), 115-127.

Ministerio de Educación de Chile. (2005). Ley 19.979 sobre Reglamento Consejos Escolares. Recuperado desde http://portales.mineduc.cl/usuarios/convivencia_escolar/doc/201103041415520. Decreto_con_Toma_de_Razon_N24_Reglamenta_Consejos_Escolares.pdf

Molina, S. (2007). Los grupos interactivos: Una práctica de las comunidades de aprendizaje para la inclusión del alumnado con discapacidad. Fondo de Tesis Universitat de Barcelona. Recuperado desde http://www.tdx.cat/bitstream/handle/10803/31986/SMR_TESIS.pdf?sequence=1

Murillo, F., Krichesky, G., Castro, A., \& Reyes, C. (2010). Liderazgo para la inclusión escolar y la justicia social. Aportaciones de la investigación. Revista Latinoamericana de Inclusión Educativa, 4(1), 169-186.

Murillo, F. J., \& Muñoz-Repiso, M. (2002) La mejora de la escuela. Un cambio de mirada. Barcelona: Octaedro.

OCDE. (2009). Informe TALIS. La creación de entornos eficaces de enseñanza y aprendizaje. Síntesis de los primeros resultados. Recuperado de http://www.ub.edu/obipd/docs/educacionp/ Informes/33.pdf

Petreñas, C. (2014). Pràctiques Dialògiques a l'Educació Secundaria. Fondo de Tesis Universitat de Barcelona. Recuperado desde http://www.tdx.cat/handle/10803/279212

Rockwell, E. (1995). De huellas, bardas y veredas, una historia cotidiana en la escuela. En E. Rockwell (Coord.), La escuela cotidiana (pp. 13-56). México: Fondo de Cultura Económica.

Sarto, M., \& Venegas, M. (Coords.). (2009). Aspectos clave de la educación inclusiva. Salamanca: Publicaciones del INICO, Colección Investigación.

Stake, R. (1999). Investigación con estudio de casos. Madrid: Morata.

Valls, R. (2000). Comunidades de aprendizaje: Una práctica educativa de aprendizaje Dialógico para la sociedad de la Información. Fondo de Tesis Universitat de Barcelona.

Vasilachis, I. (Coord.). (2009). Estrategias de investigación cualitativa. Barcelona: Gedisa.

Viera, L. (2011). Voluntariado en la escuela: Un estudio de casos dentro del proyecto comunidades de aprendizaje. Fondo de Tesis Universitat de Barcelona.

Woods, P. (1985). La Escuela por dentro. Barcelona: Paidós. 
